\title{
Inequalities among quasi-arithmetic means for continuous field of operators
}

\author{
Jadranka Mićića ${ }^{,}$Kemal Hot ${ }^{\mathrm{b}}$ \\ ${ }^{a}$ Faculty of Mechanical Engineering and Naval Architecture, University of Zagreb, Ivana Lučića 5, 10000 Zagreb, Croatia \\ ${ }^{b}$ Electrical Engineering Department, Polytechnic of Zagreb, Konavoska 2, 10000 Zagreb, Croatia
}

\begin{abstract}
In this paper we study inequalities among quasi-arithmetic means for a continuous field of self-adjoint operators, a field of positive linear mappings and continuous strictly monotone functions which induce means. We present inequalities with operator convexity and without operator convexity of appropriate functions. Also, we present a general formulation of converse inequalities in each of these cases. Furthermore, we obtain refined inequalities without operator convexity. As applications, we obtain inequalities among power means.
\end{abstract}

\section{Introduction}

We recall some notations and definitions. Let $T$ be a locally compact Hausdorff space and let $\mathcal{A}$ be a $C^{*}$-algebra of operators on some Hilbert space $H$. We say that a field $\left(x_{t}\right)_{t \in T}$ of operators in $\mathcal{A}$ is continuous if the function $t \mapsto x_{t}$ is norm continuous on $T$. If in addition $\mu$ is a Radon measure on $T$ and the function $t \mapsto\left\|x_{t}\right\|$ is integrable, then we can form the Bochner integral $\int_{T} x_{t} d \mu(t)$, which is the unique element in $\mathcal{A}$ such that

$$
\varphi\left(\int_{T} x_{t} d \mu(t)\right)=\int_{T} \varphi\left(x_{t}\right) d \mu(t)
$$

for every linear functional $\varphi$ in the norm dual $\mathcal{A}^{*}$.

Assume furthermore that there is a field $\left(\Phi_{t}\right)_{t \in T}$ of positive linear mappings $\Phi_{t}: \mathcal{A} \rightarrow \mathcal{B}$ from $\mathcal{A}$ to another $C^{*}$-algebra $\mathcal{B}$ of operators on a Hilbert space $K$. We recall that a linear mapping $\Phi_{t}: \mathcal{A} \rightarrow \mathcal{B}$ is said to be a positive mapping if $\Phi_{t}\left(x_{t}\right) \geq 0$ for all $x_{t} \geq 0$. We say that such a field is continuous if the function $t \mapsto \Phi_{t}(x)$ is continuous for every $x \in \mathcal{A}$. Let the $C^{*}$-algebras include the identity operators and the field $t \mapsto \phi_{t}\left(1_{H}\right)$ be integrable with $\int_{T} \phi_{t}\left(1_{H}\right) d \mu(t)=k 1_{K}$ for some positive scalar $k$.

Let $B(H)$ be the $C^{*}$-algebra of all bounded linear operators on a Hilbert space $H$. We define bounds of a self-adjoint operator $x \in B(H)$ by

$$
m_{x}=\inf _{\|\xi\|=1}\langle x \xi, \xi\rangle \quad \text { and } \quad M_{x}=\sup _{\|\xi\|=1}\langle x \xi, \xi\rangle
$$

2010 Mathematics Subject Classification. Primary 47A63; Secondary 47B15, 47A64

Keywords. Quasi-arithmetic mean, power mean, self-adjoint operator, positive linear mapping, convex function Received: 01 February 2011; Accepted: 21 March 2012

Communicated by Qamrul Hasan Ansari and Ljubiša D.R. Kočinac

Research supported by the Croatian Ministry of Science, Education, and Sports, under Research Grants 246-0361616-1623

Email addresses: jmicic@fsb.hr (Jadranka Mićić), khot@tvz.hr (Kemal Hot) 
for $\xi \in H$. If $\mathrm{Sp}(x)$ denotes the spectrum of $x$, then $\mathrm{Sp}(x) \subseteq\left[m_{x}, M_{x}\right]$.

For an operator $x \in B(H)$ we define operators $|x|, x^{+}, x^{-}$by

$$
|x|=\left(x^{*} x\right)^{1 / 2}, \quad x^{+}=(|x|+x) / 2, \quad x^{-}=(|x|-x) / 2 .
$$

Obviously, if $x$ is self-adjoint, then $|x|=\left(x^{2}\right)^{1 / 2}$ and $x^{+}, x^{-} \geq 0$ (called positive and negative parts of $x=x^{+}-x^{-}$.

We define a generalized quasi-arithmetic operator mean by

$$
\mathcal{M}_{\varphi} \equiv \mathcal{M}_{\varphi}(x, \Phi):=\varphi^{-1}\left(\frac{1}{k} \int_{T} \Phi_{t}\left(\varphi\left(x_{t}\right)\right) d \mu(t)\right),
$$

where $\left(x_{t}\right)_{t \in T}$ is a bounded continuous field of self-adjoint operators in a $C^{*}$-algebra $B(H)$ with spectra in $[m, M]$ for some scalars $m<M,\left(\Phi_{t}\right)_{t \in T}$ is a field of positive linear mappings $\Phi_{t}: B(H) \rightarrow B(K)$, such that $\int_{T} \Phi_{t}\left(1_{H}\right) d \mu(t)=k 1_{H}$ for some positive scalar $k$ and $\varphi \in C[m, M]$ is a strictly monotone function.

As a special case of the quasi-arithmetic mean (2) we can study the operator power mean

$$
\mathcal{M}^{[r]}(x, \Phi):= \begin{cases}\left(\int_{T} \frac{1}{k} \Phi_{t}\left(x_{t}^{r}\right) d \mu(t)\right)^{1 / r}, & r \in \mathbb{R} \backslash\{0\}, \\ \exp \left(\int_{T} \frac{1}{k} \Phi_{t}\left(\ln \left(x_{t}\right)\right) d \mu(t)\right), & r=0,\end{cases}
$$

where $\left(x_{t}\right)_{t \in T}$ is a bounded continuous field of strictly positive operators in a $C^{*}$-algebra $B(H),\left(\Phi_{t}\right)_{t \in T}$ is a field of positive linear mappings $\Phi_{t}: B(H) \rightarrow B(K)$, such that $\int_{T} \Phi_{t}\left(1_{H}\right) d \mu(t)=k 1_{K}$ for some $k>0$.

The first result on studying some inequalities such as the Jensen inequality without operator convexity is obtained in [10]. In [21] some techniques are used while one manipulates some inequalities related to continuous fields of operators. There is extensive literature devoted to quasi-arithmetic means, see, e.g. [1]-[5], [7]-[9], [19], [20].

In this paper we present inequalities with operator convexity and without operator convexity of functions which induce means. Also, we present a general formulation of converse inequalities in each of these cases. Furthermore, we obtain refined inequalities without operator convexity. As applications, we obtain inequalities among power means. These results are generalizations of our previous results obtained in a series of articles [10,11, 13, 14, 16, 17]. The interested reader will find in [12, 15] extensions of inequalities among quasi-arithmetic means in the discrete case $T=\{1, \ldots, n\}$.

\section{Inequalities with operator convexity}

We recall some classical results about quasi-arithmetic means. A result with the monotonicity is given in the next theorem.

Theorem 2.1. ([17, Theorem 2.1 and Theorem 2.3]) Let $\left(x_{t}\right)_{t \in T},\left(\Phi_{t}\right)_{t \in T}$ be as in the definition of the quasi-arithmetic mean (2). Let $\psi, \varphi \in C[m, M]$ be strictly monotone functions.

If one of the following conditions

(i) $\psi \circ \varphi^{-1}$ is operator convex and $\psi^{-1}$ is operator monotone,

(i') $\psi \circ \varphi^{-1}$ is operator concave and $-\psi^{-1}$ is operator monotone,

(ii) $\varphi^{-1}$ is operator convex and $\psi^{-1}$ is operator concave,

is satisfied then

$$
\mathcal{M}_{\varphi}(x, \Phi) \leq \mathcal{M}_{\psi}(x, \Phi) .
$$

$B u t$, if one of the following conditions

(iii) $\psi \circ \varphi^{-1}$ is operator concave and $\psi^{-1}$ is operator monotone,

(iii') $\psi \circ \varphi^{-1}$ is operator convex and $-\psi^{-1}$ is operator monotone,

(iv) $\varphi^{-1}$ is operator concave and $\psi^{-1}$ is operator convex,

is satisfied then the reverse inequality is valid in (4). 
Proof. We shall give the proof for the convenience of the reader. We will prove only the case (i).

We put $f=\psi \circ \varphi^{-1}$ in the generalized Jensen's inequality [6, Theoem 2.1]

$$
f\left(\frac{1}{k} \int_{T} \phi_{t}\left(x_{t}\right) d \mu(t)\right) \leq \frac{1}{k} \int_{T} \phi_{t}\left(f\left(x_{t}\right)\right) d \mu(t),
$$

thereafter replace $x_{t}$ with $\varphi\left(x_{t}\right)$ and finally we apply the operator monotone function $\psi^{-1}$.

Next we give inequalities complementary to the ones in Theorem 2.1. In the following theorem we give a general result. We obtain some better bounds than the ones given in [17, Theorem 3.1]).

Theorem 2.2. Let $\left(x_{t}\right)_{t \in T},\left(\Phi_{t}\right)_{t \in T}, m$ and $M$ be as in the definition of the quasi-arithmetic mean (2). Let $\psi, \varphi \in$ $C[m, M]$ be strictly monotone functions and $F:[m, M] \times[m, M] \rightarrow \mathbb{R}$ be a bounded and operator monotone function in its first variable. Let $m_{\varphi}$ and and $M_{\varphi}, m_{\varphi}<M_{\varphi}$, are bounds of the mean $\mathcal{M}_{\varphi}(x, \Phi)$.

If one of the following conditions

(i) $\psi \circ \varphi^{-1}$ is convex and $\psi^{-1}$ is operator monotone,

(i') $\psi \circ \varphi^{-1}$ is concave and $-\psi^{-1}$ is operator monotone,

is satisfied then

$$
\begin{gathered}
F\left[\mathcal{M}_{\psi}(x, \Phi), \mathcal{M}_{\varphi}(x, \Phi)\right] \\
\leq \sup _{m_{\varphi} \leq z \leq M_{\varphi}} F\left[\psi^{-1}\left(\frac{\varphi(M)-\varphi(z)}{\varphi(M)-\varphi(m)} \psi(m)+\frac{\varphi(z)-\varphi(m)}{\varphi(M)-\varphi(m)} \psi(M)\right), z\right] 1_{K} \\
\leq \sup _{m \leq z \leq M} F\left[\psi^{-1}\left(\frac{\varphi(M)-\varphi(z)}{\varphi(M)-\varphi(m)} \psi(m)+\frac{\varphi(z)-\varphi(m)}{\varphi(M)-\varphi(m)} \psi(M)\right), z\right] 1_{K} .
\end{gathered}
$$

But, if one of the following conditions

(ii) $\psi \circ \varphi^{-1}$ is concave and $\psi^{-1}$ is operator monotone,

(ii') $\psi \circ \varphi^{-1}$ is convex and $-\psi^{-1}$ is operator monotone,

is satisfied then the reverse inequality is valid in (5) with inf instead of sup.

Proof. We will prove only the case (i).

Replacing $z$ by $\varphi(z)$ and $f$ by $\psi \circ \varphi^{-1}$ in the inequality $f(z) \leq \frac{M-z}{M-m} f(m)+\frac{z-M}{M-m} f(M), z \in[m, M]$, and then using the functional calculus and operator monotonicity of $\psi^{-1}$ we obtain

$$
\mathcal{M}_{\psi} \leq \psi^{-1}\left(\frac{\varphi(M) 1_{K}-\varphi\left(\mathcal{M}_{\varphi}\right)}{\varphi(M)-\varphi(m)} \psi(m)+\frac{\varphi\left(\mathcal{M}_{\varphi}\right)-\varphi(m) 1_{K}}{\varphi(M)-\varphi(m)} \psi(M)\right) .
$$

Now, by using operator monotonicity of $F(\cdot, v)$ and $m 1_{K} \leq m_{\varphi} 1_{K} \leq \mathcal{M}_{\varphi} \leq M_{\varphi} 1_{K} \leq M 1_{K}$, we obtain

$$
\begin{aligned}
& F\left[\mathcal{M}_{\psi}, \mathcal{M}_{\varphi}\right] \\
\leq & F\left[\psi^{-1}\left(\frac{\varphi(M) 1_{K}-\varphi\left(\mathcal{M}_{\varphi}\right)}{\varphi(M)-\varphi(m)} \psi(m)+\frac{\varphi\left(\mathcal{M}_{\varphi}\right)-\varphi(m) 1_{K}}{\varphi(M)-\varphi(m)} \psi(M)\right), \mathcal{M}_{\varphi}\right] \\
\leq & \sup _{m_{\varphi} \leq z \leq M_{\varphi}} F\left[\psi^{-1}\left(\frac{\varphi(M)-\varphi(z)}{\varphi(M)-\varphi(m)} \psi(m)+\frac{\varphi(z)-\varphi(m)}{\varphi(M)-\varphi(m)} \psi(M)\right), z\right] 1_{K},
\end{aligned}
$$

which give the desired sequence of inequalities (5).

If we put $F(u, v)=u-v$ and $F(u, v)=v^{-1 / 2} u v^{-1 / 2}(v>0)$ in Theorem 2.2, then we obtain the difference and the ratio type inequalities among quasi-arithmetic means as follows. 
Corollary 2.3. Let the assumptions be as in Theorem 2.2.

If one of the conditions ( $i)$ or ( $\left.i^{\prime}\right)$ in Theorem 2.2 is satisfied then

$$
\mathcal{M}_{\psi}(x, \Phi) \leq \mathcal{M}_{\varphi}(x, \Phi)+\max _{m_{\varphi} \leq z \leq M_{\varphi}}\left\{\psi^{-1}\left(\frac{\varphi(M)-\varphi(z)}{\varphi(M)-\varphi(m)} \psi(m)+\frac{\varphi(z)-\varphi(m)}{\varphi(M)-\varphi(m)} \psi(M)\right)-z\right\} 1_{K} .
$$

If in addition $\psi^{-1}>0$ on $\left[m_{\psi}, M_{\psi}\right]$, then

$$
\mathcal{M}_{\psi}(x, \Phi) \leq \max _{m_{\varphi} \leq z \leq M_{\varphi}}\left\{\frac{\psi^{-1}\left(\frac{\varphi(M)-\varphi(z)}{\varphi(M)-\varphi(m)} \psi(m)+\frac{\varphi(z)-\varphi(m)}{\varphi(M)-\varphi(m)} \psi(M)\right)}{z}\right\} \mathcal{M}_{\varphi}(x, \Phi) .
$$

But, if one of the conditions ( $i$ ) or ( $\left.i^{\prime}\right)$ in Theorem 2.2 is satisfied then the reverse inequalities are valid in (7) and (8) with min instead of max.

If we put $\varphi(t)=t^{r}$ and $\psi(t)=t^{s}$ or $\varphi(t)=t^{s}$ and $\psi(t)=t^{r}$ in Theorem 2.1 we obtain the monotonicity of power means.

Corollary 2.4. Let $\left(x_{t}\right)_{t \in T},\left(\Phi_{t}\right)_{t \in T}$ be as in the definition of the power mean (3). It $r \leq s, r \notin(-1,1), s \notin(-1,1)$ or $1 / 2 \leq r \leq 1 \leq$ s or $r \leq-1 \leq s \leq-1 / 2$ then

$$
\mathcal{M}^{[r]}(x, \Phi) \leq \mathcal{M}^{[s]}(x, \Phi)
$$

Further, putting power functions in (8) we obtain the following ratio type inequalities among power means.

Corollary 2.5. Let $\left(x_{t}\right)_{t \in T},\left(\Phi_{t}\right)_{t \in T}$ be as in the definition of the power mean (3). Let $r, s \in \mathbb{R}, r \leq s$ and $r s \neq 0$ and $m^{[r]}$ and and $M^{[r]}, m^{[r]}<M^{[r]}$, are bounds of the mean $\mathcal{M}^{[r]}(x, \Phi)$.

(i) If $r \leq s, s \notin(-1,1), r \notin(-1,1)$ or $1 / 2 \leq r \leq 1 \leq s$ or $r \leq-1 \leq s \leq-1 / 2$ then

$$
\Delta\left(h^{[r]}, r, s\right)^{-1} \mathcal{M}^{[s]}(x, \Phi) \leq \mathcal{M}^{[r]}(x, \Phi) \leq \mathcal{M}^{[s]}(x, \Phi) .
$$

(ii) If $1 \leq s,-1<r<1 / 2, r \neq 0$ or $r \leq-1,-1 / 2<s<1, s \neq 0$ then

$$
\Delta\left(h^{[r]}, r, s\right)^{-1} \mathcal{M}^{[s]}(x, \Phi) \leq \mathcal{M}^{[r]}(x, \Phi) \leq \Delta\left(h^{[r]}, r, s\right) \mathcal{M}^{[s]}(x, \Phi) .
$$

(iii) If $-1 \leq-s \leq r \leq s \leq 1, r \neq 0$ or $-1 \leq r \leq s \leq r / 2<0$ then

$$
\Delta\left(h^{[r]}, r, 1\right)^{-1} \Delta\left(h^{[r]}, r, s\right)^{-1} \mathcal{M}^{[s]}(x, \Phi) \leq \mathcal{M}^{[r]}(x, \Phi) \leq \Delta\left(h^{[r]}, r, 1\right) \mathcal{M}^{[s]}(x, \Phi) .
$$

(iv) If $-1 / 2 \leq r / 2<s<-r \leq 1, s \neq 0$ then

$$
\Delta\left(h^{[r]}, s, 1\right)^{-1} \Delta\left(h^{[r]}, r, s\right)^{-1} \mathcal{M}^{[s]}(x, \Phi) \leq \mathcal{M}^{[r]}(x, \Phi) \leq \Delta\left(h^{[r]}, s, 1\right) \mathcal{M}^{[s]}(x, \Phi),
$$

where $h^{[r]}=M^{[r]} / h^{[r]}$ and $\Delta(h, r, s)$ is a generalized Specht ratio defined by

$$
\Delta(h, r, s):=\left\{\frac{r\left(h^{s}-h^{r}\right)}{(s-r)\left(h^{r}-1\right)}\right\}^{\frac{1}{s}}\left\{\frac{s\left(h^{r}-h^{s}\right)}{(r-s)\left(h^{s}-1\right)}\right\}^{-\frac{1}{r}}, \quad h>0 .
$$

The proof is similar to [4, Theorem 4.4] and we omit it.

In the same way, we can obtain the difference type inequality for power means.

Remark 2.6. The bounds given in Corollary 2.4 are better than the corresponding bounds given in [4, Theorem 4.4] for $s \geq 0$. We give the short proof. Since

$$
K(h, p):=\frac{h^{p}-h}{(p-1)(h-1)}\left(\frac{p-1}{p} \frac{h^{p}-1}{h^{p}-h}\right)^{p}=\max _{m \leq t \leq M} \frac{\frac{M-t}{M-m} M^{p}+\frac{t-m}{M-m} m^{p}}{t^{p}}, \quad h=\frac{M}{m}
$$


and $m \leq m^{[r]} \leq M^{[r]} \leq M$, then $K\left(h^{[r]}, p\right) \leq K(h, p)$. Taking into account that $K(h, p) \geq 1$ for $p \notin(0,1)$ it follows

$$
K\left(h^{[r]}, \frac{s}{r}\right)^{1 / s} \leq K\left(h, \frac{s}{r}\right)^{1 / s} \quad \text { for } s \geq 0 .
$$

Replacing $h^{[r]}$ and $h$ in the above inequality by $\left(h^{[r]}\right)^{r}$ and $h^{r}$, respectively, and taking into account that $\Delta(h, r, s):=K\left(h, \frac{r}{s}\right)^{1 / s}$ we obtain

$$
\Delta\left(h^{[r]}, r, s\right) \leq \Delta(h, r, s) .
$$

Then for $s \geq 0$ in the case (i) and (ii) of Corollary 2.4 we have

$$
\Delta(h, r, s)^{-1} \mathcal{M}^{[s]}(x, \Phi) \leq \Delta\left(h^{[r]}, r, s\right)^{-1} \mathcal{M}^{[s]}(x, \Phi) \leq \mathcal{M}^{[r]}(x, \Phi) \leq \mathcal{M}^{[s]}(x, \Phi)
$$

and

$$
\Delta(h, r, s)^{-1} \mathcal{M}^{[s]}(x, \Phi) \leq \Delta\left(h^{[r]}, r, s\right)^{-1} \mathcal{M}^{[s]}(x, \Phi) \leq \mathcal{M}^{[r]}(x, \Phi) \leq \Delta\left(h^{[r]}, r, s\right) \mathcal{M}^{[s]}(x, \Phi) \leq \Delta(h, r, s) \mathcal{M}^{[s]}(x, \Phi),
$$

respectively. Similarly, we can evaluate bounds in the other cases of Corollary 2.4.

\section{Inequalities without operator convexity}

In this section we give inequalities among quasi-arithmetic operator means with conditions on spectra of the operators.

In the next theorem we obtain the monotonicity of among quasi-arithmetic means without operator convexity in Theorem 2.1. This is a generalization of [10, Theorem 3].

Theorem 3.1. Let $\left(x_{t}\right)_{t \in T},\left(\Phi_{t}\right)_{t \in T}$ be as in the definition of the quasi-arithmetic mean (2). Let $m_{t}$ and $M_{t}, m_{t} \leq M_{t}$ are bounds of $x_{t}, t \in T$. Let $\varphi, \psi: I \rightarrow \mathbb{R}$ be continuous strictly monotone functions on an interval I which contains all $m_{t}, M_{t}$. Let

$$
\left(m_{\varphi}, M_{\varphi}\right) \cap\left[m_{t}, M_{t}\right]=\varnothing, \quad t \in T,
$$

where $m_{\varphi}$ and and $M_{\varphi}, m_{\varphi} \leq M_{\varphi}$, are bounds of the mean $\mathcal{M}_{\varphi}(x, \Phi)$.

If one of the following conditions

(i) $\psi \circ \varphi^{-1}$ is convex and $\psi^{-1}$ is operator monotone,

(i) $\psi \circ \varphi^{-1}$ is concave and $-\psi^{-1}$ is operator monotone,

is satisfied then

$$
\mathcal{M}_{\varphi}(x, \Phi) \leq \mathcal{M}_{\psi}(x, \Phi)
$$

But, if one of the following conditions

(ii) $\psi \circ \varphi^{-1}$ is concave and $\psi^{-1}$ is operator monotone,

(ii') $\psi \circ \varphi^{-1}$ is convex and $-\psi^{-1}$ is operator monotone

is satisfied, then the reverse inequality is valid in (9).

Proof. We will prove only the case (i).

Let $m_{\varphi}<M_{\varphi}$. Since $\operatorname{Sp}\left(\int_{T} \Phi_{t}\left(\varphi\left(x_{t}\right)\right) d \mu(t)\right) \subseteq \varphi\left(\left[m_{\varphi}, M_{\varphi}\right]\right)$ and $\psi \circ \varphi^{-1}$ is convex on $\varphi\left(\left[m_{\varphi}, M_{\varphi}\right]\right)$, then by using the functional calculus we obtain

$$
\begin{aligned}
& \psi \circ \varphi^{-1}\left(\int_{T} \frac{1}{k} \Phi_{t}\left(\varphi\left(x_{t}\right)\right) d \mu(t)\right) \\
\leq & \frac{\varphi\left(M_{\varphi}\right) 1_{K}-\int_{T} \frac{1}{k} \Phi_{t}\left(\varphi\left(x_{t}\right)\right) d \mu(t)}{\varphi\left(M_{\varphi}\right)-\varphi\left(m_{\varphi}\right)} \psi\left(m_{\varphi}\right)+\frac{\int_{T} \frac{1}{k} \Phi_{t}\left(\varphi\left(x_{t}\right)\right) d \mu(t)-\varphi\left(m_{\varphi}\right) 1_{K}}{\varphi\left(M_{\varphi}\right)-\varphi\left(m_{\varphi}\right)} \psi\left(M_{\varphi}\right) \\
= & \int_{T} \frac{1}{k} \Phi_{t}\left(\frac{\varphi\left(M_{\varphi}\right)-\varphi\left(x_{t}\right)}{\varphi\left(M_{\varphi}\right)-\varphi\left(m_{\varphi}\right)} \psi\left(m_{\varphi}\right)+\frac{\varphi\left(x_{t}\right)-\varphi\left(m_{\varphi}\right)}{\varphi\left(M_{\varphi}\right)-\varphi\left(m_{\varphi}\right)} \psi\left(M_{\varphi}\right)\right) d \mu(t) .
\end{aligned}
$$


On the other hand, since $\varphi$ is monotone, then

$$
\left(m_{\varphi}, M_{\varphi}\right) \cap\left[m_{t}, M_{t}\right]=\varnothing \quad \Longrightarrow \quad \varphi\left(\left(m_{\varphi}, M_{\varphi}\right)\right) \cap \varphi\left(\left[m_{t}, M_{t}\right]\right)=\varnothing, \quad t \in T .
$$

By using the above condition and that $m_{t} 1_{H} \leq x_{t} \leq M_{t} 1_{H}, t \in T$, then it follows

$$
\psi \circ \varphi^{-1}\left(\varphi\left(x_{t}\right)\right) \geq \frac{\varphi\left(M_{\varphi}\right) 1_{H}-\varphi\left(x_{t}\right)}{\varphi\left(M_{\varphi}\right)-\varphi\left(m_{\varphi}\right)} \psi \circ \varphi^{-1}\left(\varphi\left(m_{\varphi}\right)\right)+\frac{\varphi\left(x_{t}\right)-\varphi\left(m_{\varphi}\right) 1_{H}}{\varphi\left(M_{\varphi}\right)-\varphi\left(m_{\varphi}\right)} f\left(\varphi\left(M_{\varphi}\right)\right)
$$

for $t \in T$. Applying a positive linear mapping $\Phi_{t}$ and integrating, we obtain

$$
\begin{gathered}
\int_{T} \frac{1}{k} \Phi_{t}\left(\psi \circ \varphi^{-1}\left(\varphi\left(x_{t}\right)\right) d \mu(t)=\int_{T} \frac{1}{k} \Phi_{t}\left(\psi\left(x_{t}\right)\right) d \mu(t)\right. \\
\geq \quad \int_{T} \frac{1}{k} \Phi_{t}\left(\frac{\varphi\left(M_{\varphi}\right)-\varphi\left(x_{t}\right)}{\varphi\left(M_{\varphi}\right)-\varphi\left(m_{\varphi}\right)} \psi\left(m_{\varphi}\right)+\frac{\varphi\left(x_{t}\right)-\varphi\left(m_{\varphi}\right)}{\varphi\left(M_{\varphi}\right)-\varphi\left(m_{\varphi}\right)} \psi\left(M_{\varphi}\right)\right) d \mu(t) .
\end{gathered}
$$

Combining the two inequalities (10) and (11), and applying operator monotonicity of $\psi^{-1}$ we obtain the desired inequality (9).

In the case $m_{\varphi}=M_{\varphi}$ we use supporting line of a convex functions $\psi \circ \varphi^{-1}$ in the point $z=\varphi\left(m_{\varphi}\right)$ and similarly as above we obtain (9).

An example of the condition of spectra in the discrete case is shown in Figure 1.b).

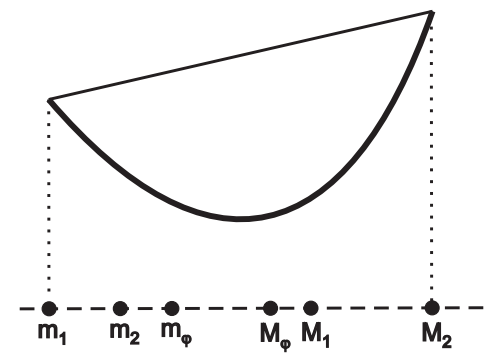

a)

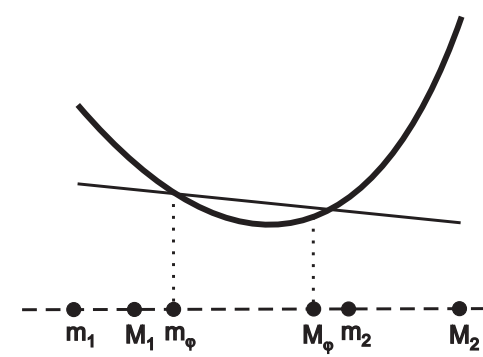

b)

Figure 1: Spectral conditions for a convex function and $T=\{1,2\}$

It is interesting to study the case when (9) holds only under the condition placed on the bounds of operators whose means we are considering. We give it in the next corollary.

Corollary 3.2. Let $\left(x_{t}\right)_{t \in T},\left(\Phi_{t}\right)_{t \in T}$ be as in the definition of the quasi-arithmetic mean (2). Let $m_{t}$ and $M_{t}, m_{t} \leq M_{t}$ are bounds of $x_{t}, t \in T$ and let $\varphi, \psi: I \rightarrow \mathbf{R}$ be continuous strictly monotone functions on an interval I which contains all $m_{t}, M_{t}$. Let

$$
\left(m_{x}, M_{x}\right) \cap\left[m_{t}, M_{t}\right]=\varnothing, \quad t \in T,
$$

where $m_{x}$ and $M_{x}, m_{x} \leq M_{x}$, are the bounds of the operator $x=\int_{T} \Phi_{t}\left(x_{t}\right) d \mu(t)$.

If one of the following conditions

(i) $\varphi$ is convex, $\varphi^{-1}$ is operator monotone, $\psi$ is concave, $\psi^{-1}$ is operator monotone,

(ii) $\varphi$ is convex, $\varphi^{-1}$ is operator monotone, $\psi$ is convex, $-\psi^{-1}$ is operator monotone,

(iii) $\varphi$ is concave, $-\varphi^{-1}$ is operator monotone, $\psi$ is convex, $-\psi^{-1}$ is operator monotone,

(iv) $\varphi$ is concave, $-\varphi^{-1}$ is operator monotone, $\psi$ is concave, $\psi^{-1}$ is operator monotone is satisfied, then

$$
\mathcal{M}_{\varphi}(x, \Phi) \leq \mathcal{M}_{\psi}(x, \Phi) .
$$

But, if one of the following conditions

$\left(i^{\prime}\right) \varphi$ is convex, $-\varphi^{-1}$ is operator monotone, $\psi$ is concave, $-\psi^{-1}$ is operator monotone, 
(ii') $\varphi$ is convex, $-\varphi^{-1}$ is operator monotone, $\psi$ is convex, $\psi^{-1}$ is operator monotone, (iii') $\varphi$ is concave, $\varphi^{-1}$ is operator monotone, $\psi$ is convex, $\psi^{-1}$ is operator monotone, (iv') $\varphi$ is concave, $\varphi^{-1}$ is operator monotone, $\psi$ is concave, $-\psi^{-1}$ is operator monotone is satisfied, then the reverse inequality is valid in (12).

Proof. We will prove only the case (i). Replacing $\varphi$ by the identity function $\mathcal{I}$ in Theorem 3.1(i) and replacing $\varphi$ by $\mathcal{I}$ and $\psi$ by $\varphi$ in Theorem 3.1(ii), we obtain (12).

Using the condition on spectra we obtain the following generalization of Theorem 2.2.

Theorem 3.3. Let $\left(x_{t}\right)_{t \in T},\left(\Phi_{t}\right)_{t \in T}$ be as in the definition of the quasi-arithmetic mean (2). Let $m_{t}$ and $M_{t}, m_{t} \leq M_{t}$ are bounds of $x_{t}, t \in T$. Let $\varphi, \psi:[m, M] \rightarrow \mathbb{R}$ be continuous strictly monotone functions, where $m=\inf _{t \in T}\left\{m_{t}\right\}$ and $M=\sup _{t \in T}\left\{M_{t}\right\}$. Let

$$
\left(m_{\varphi}, M_{\varphi}\right) \cap\left[m_{t}, M_{t}\right]=\varnothing, \quad t \in T,
$$

where $m_{\varphi}$ and $M_{\varphi}, m_{\varphi}<M_{\varphi}$, are bounds of the mean $\mathcal{M}_{\varphi}(x, \Phi)$. Let $g: \varphi\left(\left[m_{\varphi}, M_{\varphi}\right]\right) \rightarrow \mathbb{R}$ and $F:[m, M] \times V \rightarrow \mathbb{R}$ be functions such that $g\left(\varphi\left(\left[m_{\varphi}, M_{\varphi}\right]\right)\right) \subseteq V$ and $F$ be bounded and operator monotone function in its first variable. If one of the following conditions

(i) $\psi \circ \varphi^{-1}$ is convex and $\psi^{-1}$ is operator monotone,

(i') $\psi \circ \varphi^{-1}$ is concave and $-\psi^{-1}$ is operator monotone,

is satisfied then

$$
\begin{gathered}
\inf _{m_{\varphi} \leq z \leq M_{\varphi}} F\left[\psi^{-1}\left(\frac{\varphi\left(M_{\varphi}\right)-\varphi(z)}{\varphi\left(M_{\varphi}\right)-\varphi\left(m_{\varphi}\right)} \psi\left(m_{\varphi}\right)+\frac{\varphi(z)-\varphi\left(m_{\varphi}\right)}{\varphi\left(M_{\varphi}\right)-\varphi\left(m_{\varphi}\right)} \psi\left(M_{\varphi}\right)\right), g(\varphi(z))\right] 1_{K} \\
\leq F\left[\mathcal{M}_{\psi}(x, \Phi), g\left(\int_{T} \frac{1}{k} \Phi_{t}\left(\varphi\left(x_{t}\right)\right) d \mu(t)\right)\right] \\
\leq \sup _{m_{\varphi} \leq z \leq M_{\varphi}} F\left[\psi^{-1}\left(\frac{\varphi(M)-\varphi(z)}{\varphi(M)-\varphi(m)} \psi(m)+\frac{\varphi(z)-\varphi(m)}{\varphi(M)-\varphi(m)} \psi(M)\right), g(\varphi(z))\right] 1_{K} .
\end{gathered}
$$

$B u t$, if one of the following conditions

(ii) $\psi \circ \varphi^{-1}$ is concave and $\psi^{-1}$ is operator monotone,

(ii') $\psi \circ \varphi^{-1}$ is convex and $-\psi^{-1}$ is operator monotone

is satisfied, then the reverse inequalities are valid in (13) with replace sup and inf by inf and sup, respectively.

Proof. We will prove only the case (i).

By using the inequality (see (6) in the proof of Theorem 2.2)

$$
\mathcal{M}_{\psi} \leq \psi^{-1}\left(\frac{\varphi(M) 1_{K}-\varphi\left(\mathcal{M}_{\varphi}\right)}{\varphi(M)-\varphi(m)} \psi(m)+\frac{\varphi\left(\mathcal{M}_{\varphi}\right)-\varphi(m) 1_{K}}{\varphi(M)-\varphi(m)} \psi(M)\right)
$$

and operator monotonicity of $F(\cdot, v)$ we obtain RHS of (13).

Applying an operator monotone function $\psi^{-1}$ on the inequality (see (11) in the proof of Theorem 3.1)

$$
\int_{T} \frac{1}{k} \Phi_{t}\left(\frac{\varphi\left(M_{\varphi}\right)-\varphi\left(x_{t}\right)}{\varphi\left(M_{\varphi}\right)-\varphi\left(m_{\varphi}\right)} \psi\left(m_{\varphi}\right)+\frac{\varphi\left(x_{t}\right)-\varphi\left(m_{\varphi}\right)}{\varphi\left(M_{\varphi}\right)-\varphi\left(m_{\varphi}\right)} \psi\left(M_{\varphi}\right)\right) d \mu(t) \leq \psi\left(\mathcal{M}_{\psi}\right)
$$

we obtain

$$
\psi^{-1}\left(\frac{\varphi\left(M_{\varphi}\right) 1_{K}-\varphi\left(\mathcal{M}_{\varphi}\right)}{\varphi\left(M_{\varphi}\right)-\varphi\left(m_{\varphi}\right)} \psi\left(m_{\varphi}\right)+\frac{\varphi\left(\mathcal{M}_{\varphi}\right)-\varphi\left(m_{\varphi}\right) 1_{K}}{\varphi\left(M_{\varphi}\right)-\varphi\left(m_{\varphi}\right)} \psi\left(M_{\varphi}\right)\right) \leq \mathcal{M}_{\psi} .
$$

By using operator monotonicity of $F(\cdot, v)$ we obtain LHS of (13).

Putting $g \equiv \varphi^{-1}$ and $F(u, v)=u-\alpha v$ or $F(u, v)=v^{-1 / 2} u v^{-1 / 2}$, we obtain the next corollary. 
Corollary 3.4. Let $\left(x_{t}\right)_{t \in T},\left(\Phi_{t}\right)_{t \in T}$ be as in the definition of the quasi-arithmetic mean (2). Let $m_{t}$ and $M_{t}, m_{t} \leq M_{t}$ are bounds of $x_{t}, t \in T$ and $\varphi, \psi:[m, M] \rightarrow \mathbb{R}$ be continuous strictly monotone functions, where $m=\inf _{t \in T}\left\{m_{t}\right\}$ and $M=\sup _{t \in T}\left\{M_{t}\right\}$. Let

$$
\left(m_{\varphi}, M_{\varphi}\right) \cap\left[m_{t}, M_{t}\right]=\varnothing, \quad t \in T,
$$

where $m_{\varphi}$ and $M_{\varphi}, m_{\varphi}<M_{\varphi}$, are bounds of the mean $\mathcal{M}_{\varphi}(x, \Phi)$.

If one of the following conditions

(i) $\psi \circ \varphi^{-1}$ is convex and $\psi^{-1}$ is operator monotone,

(i) $\psi \circ \varphi^{-1}$ is concave and $-\psi^{-1}$ is operator monotone,

is satisfied then for any real number $\alpha$ the following sequence of inequalities

$$
\begin{gathered}
\min _{m_{\varphi} \leq z \leq M_{\varphi}}\left\{\psi^{-1}\left(\frac{\varphi\left(M_{\varphi}\right)-\varphi(z)}{\varphi\left(M_{\varphi}\right)-\varphi\left(m_{\varphi}\right)} \psi\left(m_{\varphi}\right)+\frac{\varphi(z)-\varphi\left(m_{\varphi}\right)}{\varphi\left(M_{\varphi}\right)-\varphi\left(m_{\varphi}\right)} \psi\left(M_{\varphi}\right)\right)-\alpha z\right\} 1_{K} \\
\quad \leq \mathcal{M}_{\psi}(x, \Phi)-\alpha \mathcal{M}_{\varphi}(x, \Phi) \\
\leq \max _{m_{\varphi} \leq z \leq M_{\varphi}}\left\{\psi^{-1}\left(\frac{\varphi(M)-\varphi(z)}{\varphi(M)-\varphi(m)} \psi(m)+\frac{\varphi(z)-\varphi(m)}{\varphi(M)-\varphi(m)} \psi(M)\right)-\alpha z\right\} 1_{K} .
\end{gathered}
$$

If in addition $\psi^{-1}>0$ on $\left[m_{\psi}, M_{\psi}\right]$, then

$$
\begin{gathered}
\min _{m_{\varphi} \leq z \leq M_{\varphi}}\left\{\frac{\psi^{-1}\left(\frac{\varphi\left(M_{\varphi}\right)-\varphi(z)}{\varphi(M \varphi)-\varphi(m \varphi)} \psi\left(m_{\varphi}\right)+\frac{\varphi(z)-\varphi\left(m_{\varphi}\right)}{\varphi(M \varphi)-\varphi(m \varphi)} \psi\left(M_{\varphi}\right)\right)}{z}\right\} \mathcal{M}_{\varphi}(x, \Phi) \\
\leq \mathcal{M}_{\psi}(x, \Phi) \leq \max _{m_{\varphi} \leq z \leq M_{\varphi}}\left\{\frac{\psi^{-1}\left(\frac{\varphi(M)-\varphi(z)}{\varphi(M)-\varphi(m)} \psi(m)+\frac{\varphi(z)-\varphi(m)}{\varphi(M)-\varphi(m)} \psi(M)\right)}{z}\right\} \mathcal{M}_{\varphi}(x, \Phi) .
\end{gathered}
$$

But, if one of the following conditions

(ii) $\psi \circ \varphi^{-1}$ is concave and $\psi^{-1}$ is operator monotone,

(ii') $\psi \circ \varphi^{-1}$ is convex and $-\psi^{-1}$ is operator monotone

is satisfied, then for any real number $\alpha$ the reverse inequalities are valid in (14) and (15) (for $\psi^{-1}>0$ ) with replace sup and inf by inf and sup, respectively.

If we put $\varphi(t)=t^{r}$ and $\psi(t)=t^{s}$ in Theorem 3.1 and Corollary 3.4 we obtain the order among power means as follows. These results are a generalization of [10, Corollary 7] and [11, Theorem 1].

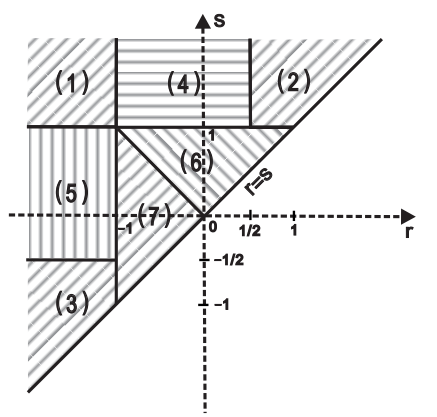

a) WITHOUT CONDITION ON SPECTRA:

$\Delta=1$, for $r, s$ in (1), (2), (3)

$\Delta=\mathrm{K}\left(\mathrm{h}^{\mathrm{s}}, r / s\right)^{1 / r}$, for $r, s$ in (4), (5)

$\Delta=\mathrm{K}(\mathrm{h}, r)^{-1 / r}$, for $r, s$ in (6)

$\Delta=\mathrm{K}(\mathrm{h}, s)^{-1 / s}$, for $r, s$ in (7)

b) WITH CONDITION ON SPECTRA

$\Delta=1$, for $r, s$ in (1), (2), (4) or (1), (3), (5)

$\Delta=\mathrm{K}\left(\mathrm{h}^{[\mathrm{r}]}, s\right)^{-1 / s}$ or $\Delta=\mathrm{K}\left(\mathrm{h}^{[\mathrm{s}]}, r\right)^{-1 / r}$, for $r, s$ in (6), (7)

Figure 2: Regions for the inequality $\mathcal{M}^{[r]}(\mathbf{A}, \Phi) \leq \Delta \mathcal{M}^{[s]}(\mathbf{A}, \Phi)$

Corollary 3.5. Let $\left(x_{t}\right)_{t \in T},\left(\Phi_{t}\right)_{t \in T}$ be as in the definition of the power mean (3). Let $m_{t}$ and $M_{t}, 0<m_{t} \leq M_{t}$ be the bounds of $x_{t}, t \in T$.

If one of the following conditions 
(i) $r \leq s, s \geq 1$ or $r \leq s \leq-1$ (Figure 2.b(1)-(4)) and

$$
\left(m^{[r]}, M^{[r]}\right) \cap\left[m_{t}, M_{t}\right]=\varnothing, \quad t \in T,
$$

where $m^{[r]}$ and $M^{[r]}, m^{[r]} \leq M^{[r]}$ are the bounds of $\mathcal{M}^{[r]}(x, \Phi)$,

(ii) $r \leq s, r \leq-1$ or $1 \leq r \leq s$ (Figure 2.b (1)-(3),(5)) and

$$
\left(m^{[s]}, M^{[s]}\right) \cap\left[m_{t}, M_{t}\right]=\varnothing, \quad t \in T,
$$

where $m^{[s]}$ and $M^{[s]}, m^{[s]} \leq M^{[s]}$ are the bounds of $\mathcal{M}^{[s]}(x, \Phi)$

is satisfied, then

$$
\mathcal{M}^{[r]}(x, \Phi) \leq \mathcal{M}^{[s]}(x, \Phi) .
$$

(iii) If $r, s \in(-1,1), r \leq s$ (Figure 2. (6),(7)) and (16) is valid, then

$$
\mathcal{M}^{[r]}(x, \Phi) \leq \Delta\left(h^{[r]}, s\right) \mathcal{M}^{[s]}(x, \Phi), \quad h^{[r]}=M^{[r]} / m^{[r]} .
$$

(iv) If $r, s \in(-1,1), r \leq s$ (Figure 2. (6),(7)) and (17) is valid, then

$$
\mathcal{M}^{[r]}(x, \Phi) \leq \Delta\left(h^{[s]}, r\right) \mathcal{M}^{[s]}(x, \Phi), \quad h^{[s]}=M^{[s]} / m^{[s]} .
$$

A constant $\Delta(h, p) \equiv \Delta(h, p, 1)$ is defined as follows

$$
\Delta(h, p):= \begin{cases}\frac{p\left(h-h^{p}\right)}{(1-p)\left(h^{p}-1\right)}\left(\frac{(p-1)(h-1)}{h^{p}-h}\right)^{\frac{1}{p}}, & h \neq 1 \text { and } p \neq 0, \\ \frac{(h-1) h \frac{1}{h-1}}{e \ln h}, & h \neq 1 \text { and } p=0, \\ 1, & h=1 \text { and } p \in \mathbb{R},\end{cases}
$$

where $h=M / m$.

Proof. The proof is quite similar to the ones [10, Corollary 7] and [11, Theorem 1]. We give it for the convenience of the reader.

(i): We put $\varphi(t)=t^{r}$ and $\psi(t)=t^{s}$ for $t>0$.

Then $\psi \circ \varphi^{-1}(t)=t^{s / r}$ is concave for $r \leq s, s \leq 0$ and $r \neq 0$. Since $-\psi^{-1}(t)=-t^{1 / s}$ is operator monotone for $s \leq-1$ and $\left(m^{[r]}, M^{[r]}\right) \cap\left[m_{t}, M_{t}\right]=\varnothing$ is satisfied, then by applying Theorem 3.1-(i') we obtain (18) for $r \leq s \leq-1$.

But, $\psi \circ \varphi^{-1}(t)=t^{s / r}$ is convex for $r \leq s, s \geq 0$ and $r \neq 0$. Since $\psi^{-1}(t)=t^{1 / s}$ is operator monotone for $s \geq 1$, then by applying Theorem 3.1-(i) we obtain (18) for $r \leq s, s \geq 1, r \neq 0$.

If $r=0$ and $s \geq 1$, we put $\varphi(t)=\ln t$ and $\psi(t)=t^{s}, t>0$. Since $\psi \circ \varphi^{-1}(t)=\exp (s t)$ is convex, then similarly as above we obtain the desired inequality.

(ii): We put $\varphi(t)=t^{s}$ and $\psi(t)=t^{r}$ for $t>0$ and we use the same technique as in the case (i).

(iii)-a): Let $m^{[r]}<M^{[r]}$. Suppose that $0<r \leq s \leq 1$. Then

$$
\left(m^{[r]}, M^{[r]}\right) \cap\left[m_{t}, M_{t}\right]=\varnothing \Longrightarrow\left(\left(m^{[r]}\right)^{r},\left(M^{[r]}\right)^{r}\right) \cap\left[m_{t}^{r}, M_{t}^{r}\right]=\varnothing, \quad t \in T .
$$

Putting $f(t)=t^{s / r}$, which is convex, in the two inequalities (10) and (11), we obtain

$$
\left(\int_{T} \Phi_{t}\left(x_{t}^{r}\right) d \mu(t)\right)^{s / r} \leq \int_{T} \Phi_{t}\left(x_{t}^{s}\right) d \mu(t) .
$$


Now, applying results about the function order (see [18, Corollary 6.5]) for the power function $t \mapsto t^{p}$, $p=\frac{1}{s} \geq 1$, we obtain the desired inequality (19).

the remaining the remaining combinations for $r, s \in(-1,1)$ we use the same technique as above.

(iii)-b): If $m^{[r]}=M^{[r]}$, we put $m^{[r]} \rightarrow M^{[r]}$ in inequalities (iii)-a), and we use that $\lim _{h \rightarrow 1} C(h, p)=1$ for $p \neq 1$ and $\lim _{h \rightarrow 1} C(h, 0)=1$.

(iv): We use the same technique as in the case (iii).

\section{Refined inequalities}

In this section we give a refinement of the inequality (9).

For convenience we introduce the following denotations:

$$
\begin{aligned}
\delta_{\varphi, \psi}(m, M) & =\psi(m)+\psi(M)-2 \psi \circ \varphi^{-1}\left(\frac{\varphi(m)+\varphi(M)}{2}\right), \\
\widetilde{x}_{\varphi}(m, M) & =\frac{1}{2} 1_{K}-\frac{1}{|\varphi(M)-\varphi(m)|}\left|\int_{T} \frac{1}{k} \Phi_{t}\left(\varphi\left(x_{t}\right)\right) d \mu(t)-\frac{\varphi(M)+\varphi(m)}{2} 1_{K}\right|,
\end{aligned}
$$

where $\left(x_{t}\right)_{t \in T}$ is a bounded continuous field of operators in a $C^{*}$-algebra $B(H)$ with spectra in $I,\left(\Phi_{t}\right)_{t \in T}$ is a field of positive linear mappings $\Phi_{t}: B(H) \rightarrow B(K)$, such that $\int_{T} \Phi_{t}\left(1_{K}\right) d \mu(t)=k 1_{K}$ for some positive scalar $\mathrm{k}, \varphi, \psi: I \rightarrow \mathbb{R}$ are continuous strictly monotone functions and $m, M \in I, m<M$. Of course, we include implicitly that $\widetilde{x}_{\varphi}(m, M) \equiv \widetilde{x}_{\varphi, x}(m, M)$, where $x=\int_{T} \Phi_{t}\left(\varphi\left(x_{t}\right)\right) d \mu(t)$.

To obtain our main result we need the following result.

Lemma 4.1. ([14, Lemma 2]) Let $x$ be a self-adjoint element in $B(H)$ with $\mathrm{Sp}(x) \subseteq[m, M]$, for some scalars $m<M$. Then

$$
f(x) \leq \frac{M 1_{H}-x}{M-m} f(m)+\frac{x-m 1_{H}}{M-m} f(M)-\delta_{f} \widetilde{x}
$$

holds for every continuous convex function $f:[m, M] \rightarrow \mathbb{R}$, where

$$
\delta_{f}=f(m)+f(M)-2 f\left(\frac{m+M}{2}\right) \text { and } \tilde{x}=\frac{1}{2} 1_{H}-\frac{1}{M-m}\left|x-\frac{m+M}{2} 1_{H}\right| .
$$

If $f$ is concave, then the reverse inequality is valid in (22).

The next theorem is a generalization of [14, Theorem 7].

Theorem 4.2. Let $\left(x_{t}\right)_{t \in T}$ and $\left(\Phi_{t}\right)_{t \in T}$ be as in the definition of the quasi-arithmetic mean (2). Let $m_{t}$ and $M_{t}, m_{t} \leq M_{t}$ be the bounds of $x_{t}, t \in T$. Let $\varphi, \psi: I \rightarrow \mathbb{R}$ be continuous strictly monotone functions on an interval I which contains all $m_{t}, M_{t}$. Let

$$
\left(m_{\varphi}, M_{\varphi}\right) \cap\left[m_{t}, M_{t}\right]=\varnothing, \quad t \in T, \quad \text { and } \quad m<M,
$$

where $m_{\varphi}$ and and $M_{\varphi}, m_{\varphi} \leq M_{\varphi}$, are bounds of the mean $\mathcal{M}_{\varphi}(x, \Phi)$ and $m=\sup \left\{M_{t}: M_{t} \leq m_{\varphi}, t \in T\right\}, M=$ $\inf \left\{m_{t}: m_{t} \geq M_{\varphi}, t \in T\right\}$.

(i) If $\psi \circ \varphi^{-1}$ is convex and $\psi^{-1}$ is operator monotone, then

$$
\mathcal{M}_{\varphi}(x, \Phi) \leq \psi^{-1}\left(\int_{T} \frac{1}{k} \Phi_{t}\left(\psi\left(x_{t}\right)\right) d \mu(t)-\delta_{\varphi, \psi} \widetilde{x}_{\varphi}\right) \leq \mathcal{M}_{\psi}(x, \Phi),
$$

where $\delta_{\varphi, \psi} \geq 0$ and $\widetilde{x}_{\varphi} \geq 0$.

(i') If $\psi \circ \varphi^{-1}$ is convex and $-\psi^{-1}$ is operator monotone, then the reverse inequality is valid in (23), where $\delta_{\varphi, \psi} \geq 0$ and $\widetilde{x}_{\varphi} \geq 0$. 
(ii) If $\psi \circ \varphi^{-1}$ is concave and $-\psi^{-1}$ is operator monotone, then (23) holds, where $\delta_{\varphi, \psi} \leq 0$ and $\widetilde{x}_{\varphi} \geq 0$.

(ii') If $\psi \circ \varphi^{-1}$ is concave and $\psi^{-1}$ is operator monotone, then the reverse inequality is valid in (23), where $\delta_{\varphi, \psi} \leq 0$ and $\widetilde{x}_{\varphi} \geq 0$.

In all the above cases, we assume that $\delta_{\varphi, \psi} \equiv \delta_{\varphi, \psi}(\bar{m}, \bar{M}), \widetilde{x}_{\varphi} \equiv \widetilde{x}_{\varphi}(\bar{m}, \bar{M})$ are defined by (21) and $\bar{m} \in\left[m, m_{\varphi}\right]$, $\bar{M} \in\left[M_{\varphi}, M\right], \bar{m}<\bar{M}$, are arbitrary numbers.

Proof. The proof of Theorem 4.2 is similar to the ones of Theorem 3.1, but in addition we use Lemma C.

We only prove the case (i). Putting a convex function $f \equiv \psi \circ \varphi^{-1}($ on $\varphi([\bar{m}, \bar{M}]))$ and $x=\int_{T} \frac{1}{k} \Phi_{t}\left(\varphi\left(x_{t}\right)\right) d \mu(t)\left(=\varphi\left(\mathcal{M}_{\varphi}\right)\right)$ in (22) we obtain

$$
\psi\left(\mathcal{M}_{\varphi}\right) \leq \frac{\varphi(\bar{M}) 1_{K}-\varphi\left(\mathcal{M}_{\varphi}\right)}{\varphi(\bar{M})-\varphi(\bar{m})} \psi(\bar{m})+\frac{\varphi\left(\mathcal{M}_{\varphi}\right)-\varphi(\bar{m}) 1_{K}}{\varphi(\bar{M})-\varphi(\bar{m})} \psi(\bar{M})-\delta_{\varphi, \psi} \widetilde{x}_{\varphi}
$$

where $\delta_{\varphi, \psi}$ and $\widetilde{x}_{\varphi}$ are defined by (21).

On the other hand, since

$$
\begin{aligned}
\left(m_{\varphi}, M_{\varphi}\right) \cap\left[m_{t}, M_{t}\right]=\varnothing & \Longrightarrow(\bar{m}, \bar{M}) \cap\left[m_{t}, M_{t}\right]=\varnothing \Longrightarrow \\
& \Longrightarrow \varphi((\bar{m}, \bar{M})) \cap \varphi\left(\left[m_{t}, M_{t}\right]\right)=\varnothing, \quad t \in T,
\end{aligned}
$$

then

$$
\psi\left(x_{t}\right)=\psi \circ \varphi^{-1}\left(\varphi\left(x_{t}\right)\right) \geq \frac{\varphi(\bar{M}) 1_{H}-\varphi\left(x_{t}\right)}{\varphi(\bar{M})-\varphi(\bar{m})} \psi(\bar{m})+\frac{\varphi\left(x_{t}\right)-\varphi(\bar{m}) 1_{H}}{\varphi(\bar{M})-\varphi(\bar{m})} \psi(\bar{M}) .
$$

Applying a positive linear mapping $\Phi_{t}$, integrating and adding $-\delta_{\varphi, \psi} \widetilde{x}_{\varphi}$, we obtain

$$
\begin{aligned}
& \int_{T} \frac{1}{k} \Phi_{i}\left(\psi\left(x_{t}\right)\right) d \mu(t)-\delta_{\varphi, \psi} \widetilde{x}_{\varphi} \\
\geq & \frac{\varphi(\bar{M}) 1_{K}-\varphi\left(\mathcal{M}_{\varphi}\right)}{\varphi(\bar{M})-\varphi(\bar{m})} \psi(\bar{m})+\frac{\varphi\left(\mathcal{M}_{\varphi}\right)-\varphi(\bar{m}) 1_{K}}{\varphi(\bar{M})-\varphi(\bar{m})} \psi(\bar{M})-\delta_{\varphi, \psi} \widetilde{x}_{\varphi} .
\end{aligned}
$$

Combining the two inequalities (24) and (25), and considering that $\delta_{\varphi, \psi} \geq 0$ and $\widetilde{x}_{\varphi} \geq 0$ holds, we obtain

$$
\psi\left(\mathcal{M}_{\varphi}\right) \leq \psi\left(\mathcal{M}_{\psi}\right)-\delta_{\varphi, \psi} \widetilde{x}_{\varphi} \leq \psi\left(\mathcal{M}_{\psi}\right) .
$$

Applying the operator monotone function $\psi^{-1}$ we obtain (23).

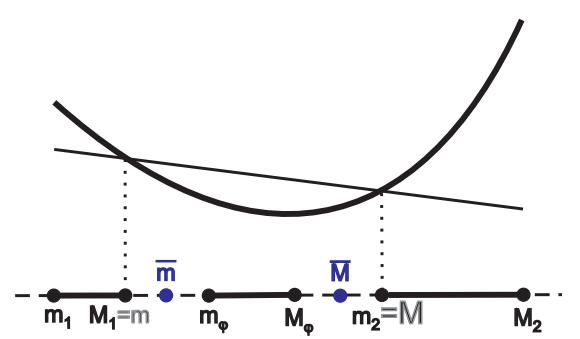

$$
\begin{aligned}
& \begin{aligned}
\varphi^{-1}\left(\Phi_{1}\left(\varphi\left(\mathrm{x}_{1}\right)\right)+\Phi_{2}\left(\varphi\left(\mathrm{x}_{2}\right)\right)\right) & \leq \psi^{-1}\left(\Phi_{1}\left(\psi\left(\mathrm{x}_{1}\right)\right)+\Phi_{2}\left(\psi\left(\mathrm{x}_{2}\right)\right)-\delta_{\varphi, \psi} \tilde{\mathrm{x}}_{\varphi}\right) \\
& \leq \psi^{-1}\left(\Phi_{1}\left(\psi\left(\mathrm{x}_{1}\right)\right)+\Phi_{2}\left(\psi\left(\mathrm{x}_{2}\right)\right)\right), \\
\text { whe re } &
\end{aligned} \\
& \delta_{\varphi, \psi}=\psi(\overline{\mathrm{m}})+\psi(\overline{\mathrm{M}})-2 \psi \circ \varphi^{-1}\left(\frac{\varphi(\overline{\mathrm{M}})-\varphi(\overline{\mathrm{m}})}{2}\right), \\
& \tilde{\mathrm{x}}_{\varphi}=\frac{1}{2} 1_{\mathrm{K}}-\frac{1}{|\varphi(\overline{\mathrm{M}})-\varphi(\overline{\mathrm{m}})|}\left|\Phi_{1}\left(\varphi\left(\mathrm{x}_{1}\right)\right)+\Phi_{2}\left(\varphi\left(\mathrm{x}_{2}\right)\right)-\frac{\varphi(\overline{\mathrm{M}})+\varphi(\overline{\mathrm{m}})}{2} 1_{\mathrm{K}}\right|
\end{aligned}
$$

Figure 3: Refinement for two operators and a convex function $\psi \circ \varphi^{-1}$

Example 4.3. We give a small example of a refined inequality among means for the matrix cases and $T=\{1,2\}$ (see Figure 3).

We put $\varphi(t)=t^{1 / 3}, \psi(t)=t^{5}$ and we define $\Phi_{1}, \Phi_{2}: M_{2}(\mathbb{C}) \rightarrow M_{2}(\mathbb{C})$ by $\Phi_{1}(B)=\Phi_{2}(B)=\frac{1}{2} B$ for $B \in M_{2}(\mathbb{C})$. 


$$
\begin{aligned}
& \text { If } x_{1}=\left(\begin{array}{cc}
13 & 8 \\
8 & 5
\end{array}\right) \text { and } x_{2}=125\left(\begin{array}{ll}
1 & 0 \\
0 & 1
\end{array}\right) \text {, then } \\
& \qquad \begin{aligned}
\mathcal{M}^{[1 / 3]}=\left(\frac{1}{2} \sqrt[3]{x_{1}}+\frac{1}{2} \sqrt[3]{x_{2}}\right)^{3}=\left(\begin{array}{cc}
45.375 & 16 \\
16 & 29.375
\end{array}\right), \\
\mathcal{M}^{[5]}=\sqrt[5]{\frac{1}{2} x_{1}^{5}+\frac{1}{2} x_{2}^{5}}=\left(\begin{array}{cc}
108.81978 & 0.00059 \\
0.00059 & 108.81919
\end{array}\right)
\end{aligned}
\end{aligned}
$$

and $m_{1}=0.05573, M_{1}=17.9443, m_{2}=M_{2}=125, m_{1 / 3}=19.4865$ and $M_{1 / 3}=55.2635$ (rounded to five decimal places).

It follows that $m=17.94427, M=125, \bar{m} \in[17.9443,19.4865]$ and $\bar{M} \in[55.2635,125]$.

Let $\bar{m}=17.94427, \bar{M}=125$.

Then we get $\delta_{1 / 3,5}=2.94885 \times 10^{10}, \widetilde{x}_{1 / 3}=\left(\begin{array}{ll}0.37027 & 0.20991 \\ 0.20991 & 0.16036\end{array}\right)$ and

$$
\mathcal{M}^{[1 / 3]}<\sqrt[5]{\frac{1}{2} x_{1}^{5}+\frac{1}{2} x_{2}^{5}-\delta_{1 / 3,5} \widetilde{x}_{1 / 3}}=\left(\begin{array}{cc}
69.70109 & -23.36045 \\
-23.36045 & 93.06154
\end{array}\right)<\mathcal{M}^{[5]}
$$

Putting the identity function in Theorem 4.2 we obtain another refinement of (9).

Corollary 4.4. Let $\left(x_{t}\right)_{t \in T}$ and $\left(\Phi_{t}\right)_{t \in T}$ be as in the definition of the quasi-arithmetic mean (2). Let $m_{t}$ and $M_{t}, m_{t} \leq M_{t}$ be the bounds of $x_{t}, t \in T$. Let $\varphi, \psi: I \rightarrow \mathbb{R}$ be continuous strictly monotone functions on an interval I which contains all $m_{t}, M_{t}$, such that $\varphi^{-1}$ is convex, $\psi^{-1}$ is concave. We denote $\mathcal{I}$ the identity function on $I$.

If

$$
\begin{aligned}
& \left(m_{\varphi}, M_{\varphi}\right) \cap\left[m_{t}, M_{t}\right]=\varnothing, \quad t \in T, \quad \text { and } \quad m_{[\varphi]}<M_{[\varphi]}, \\
& \left(m_{\psi}, M_{\psi}\right) \cap\left[m_{t}, M_{t}\right]=\varnothing, \quad t \in T, \quad \text { and } \quad m_{[\psi]}<M_{[\psi]}
\end{aligned}
$$

are valid, where $m_{\varphi}$ and $M_{\varphi}, m_{\varphi} \leq M_{\varphi}$ are the bounds of $\mathcal{M}_{\varphi}(x, \Phi)$ and $m_{[\varphi]}=\sup \left\{M_{t}: M_{t} \leq m_{\varphi}, t \in T\right\}$, $M_{[\varphi]}=\inf \left\{m_{t}: m_{t} \geq M_{\varphi}, t \in T\right\}$ and analogously for $\psi$, then the following inequality

$$
\mathcal{M}_{\varphi}(x, \Phi) \leq \mathcal{M}_{\varphi}(x, \Phi)+\Delta_{\varphi, \psi}(\bar{m}, \bar{M}, \overline{\bar{m}}, \overline{\bar{M}}) \leq \mathcal{M}_{\psi}(x, \Phi)
$$

for every $\bar{m} \in\left[m_{[\varphi]}, m_{\varphi}\right], \bar{M} \in\left[M_{\varphi}, M_{[\varphi]}\right], \bar{m}<\bar{M}$ and every $\overline{\bar{m}} \in\left[m_{[\psi], m_{\psi}}\right], \overline{\bar{M}} \in\left[M_{\psi}, M_{[\psi]}\right], \overline{\bar{m}}<\overline{\bar{M}}$, where

$$
\Delta_{\varphi, \psi}(\bar{m}, \bar{M}, \overline{\bar{m}}, \overline{\bar{M}})=\delta_{\varphi, I}(\bar{m}, \bar{M}) \widetilde{x}_{\varphi}(\bar{m}, \bar{M})-\delta_{\psi, I}(\overline{\bar{m}}, \overline{\bar{M}}) \widetilde{x}_{\psi}(\overline{\bar{m}}, \overline{\bar{M}}) \geq 0
$$

and $\delta_{\varphi, I}, \delta_{\psi, I}, \widetilde{x}_{\varphi}$ and $\widetilde{x}_{\psi}$ are defined by (21).

Proof. Putting $\psi=\mathcal{I}$ in Theorem 4.2 (i), we obtain

$$
M_{\varphi}(x, \Phi) \leq M_{\mathcal{I}}(x, \Phi)-\delta_{\varphi, \mathcal{I}}(\bar{m}, \bar{M}) \widetilde{x}_{\varphi}(\bar{m}, \bar{M}) \leq M_{\mathcal{I}}(x, \Phi),
$$

where $\delta_{\varphi, I}(\bar{m}, \bar{M}) \geq 0$. Putting $\psi=\mathcal{I}$ and replacing $\varphi$ by $\psi$ in Theorem 4.2 (ii'), we obtain

$$
M_{\psi}(x, \Phi) \geq M_{\mathcal{I}}(x, \Phi)-\delta_{\psi, \mathcal{I}}(\overline{\bar{m}}, \overline{\bar{M}}) \widetilde{x}_{\psi}(\overline{\bar{m}}, \overline{\bar{M}}) \geq M_{\mathcal{I}}(x, \Phi),
$$

where $\delta_{\psi, I}(\overline{\bar{m}}, \overline{\bar{M}}) \leq 0$.

Adding

$$
\delta_{\varphi, \mathcal{I}}(\bar{m}, \bar{M}) \widetilde{x}_{\varphi}(\bar{m}, \bar{M})-\delta_{\psi, \mathcal{I}}(\overline{\bar{m}}, \overline{\bar{M}}) \widetilde{x}_{\psi}(\overline{\bar{m}}, \overline{\bar{M}})
$$

in (27) and taking into account (28), we obtain (26). 
As an application of results given in Theorem 4.2 we study a refinement of inequality (18).

For convenience we introduce denotations as a special case of (21) as follows

$$
\begin{aligned}
\delta_{r, s}(m, M) & = \begin{cases}m^{s}+M^{s}-2\left(\frac{m^{r}+M^{r}}{2}\right)^{s / r}, & r \neq 0, \\
m^{s}+M^{s}-2(m M)^{s / 2}, & r=0,\end{cases} \\
\widetilde{x}_{r}(m, M) & = \begin{cases}\frac{1}{2} 1_{K}-\frac{1}{\left|M^{r}-m^{r}\right|}\left|\int_{T} \Phi_{t}\left(x_{t}^{r}\right) d \mu-\frac{M^{r}+m^{r}}{2} 1_{K}\right|, & r \neq 0, \\
\frac{1}{2} 1_{K}-\left|\ln \left(\frac{M}{m}\right)\right|^{-1}\left|\int_{T} \Phi_{t}\left(\ln x_{t}\right) d \mu-\ln \sqrt{M m} 1_{K}\right|, & r=0,\end{cases}
\end{aligned}
$$

where $m, M \in \mathbb{R}, 0<m<M$ and $r, s \in \mathbb{R}, r \leq s$. Of course, we include implicitly that $\widetilde{x}_{r}(m, M) \equiv \widetilde{x}_{r, x}(m, M)$, where $x=\int_{T} \Phi_{t}\left(x_{t}^{r}\right) d \mu$ for $r \neq 0$ and $x=\int_{T} \Phi_{t}\left(\ln x_{t}\right) d \mu$ for $r=0$.

The next corollary is a generalization of [10, Corollary 12].

Corollary 4.5. Let $\left(x_{t}\right)_{t \in T},\left(\Phi_{t}\right)_{t \in T}$ be as in the definition of the power mean (3). Let $m_{t}$ and $M_{t}, 0<m_{t} \leq M_{t}$ be the bounds of $x_{t}, t \in T$.

(i) If $r \leq s, s \geq 1$ or $r \leq s \leq-1$

$$
\left(m^{[r]}, M^{[r]}\right) \cap\left[m_{t}, M_{t}\right]=\varnothing, \quad t \in T, \quad \text { and } \quad m<M,
$$

where $m^{[r]}$ and $M^{[r]}, m^{[r]} \leq M^{[r]}$ are the bounds of $\mathcal{M}^{[r]}(x, \Phi)$ and $m=\sup \left\{M_{t}: M_{t} \leq m^{[r]}, t \in T\right\}, M=\inf \left\{m_{t}: m_{t} \geq M^{[r]}, t \in T\right\}$, then

$$
\mathcal{M}^{[r]}(x, \Phi) \leq\left(\int_{T} \Phi_{t}\left(x_{t}^{s}\right) d \mu-\delta_{r, s} \widetilde{x}_{r}\right)^{1 / s} \leq \mathcal{M}^{[s]}(x, \Phi),
$$

where $\delta_{r, s} \geq 0$ for $s \geq 1, \delta_{r, s} \leq 0$ for $s \leq-1$ and $\widetilde{x}_{r} \geq 0$. Here we assume that $\delta_{r, s} \equiv \delta_{r, s}(\bar{m}, \bar{M}), \widetilde{x}_{r} \equiv \widetilde{x}_{r}(\bar{m}, \bar{M})$ are defined by (29) and $\bar{m} \in\left[m, m^{[r]}\right], \bar{M} \in\left[M^{[r]}, M\right], \bar{m}<\bar{M}$, are arbitrary numbers.

(ii) If $r \leq s, r \leq-1$ or $1 \leq r \leq s$,

$$
\left(m^{[s]}, M^{[s]}\right) \cap\left[m_{t}, M_{t}\right]=\varnothing, \quad t \in T, \quad \text { and } \quad m<M,
$$

where $m^{[s]}$ and $M^{[s]}, m^{[s]} \leq M^{[s]}$ are the bounds of $\mathcal{M}^{[s]}(x, \Phi)$ and $m=\sup \left\{M_{t}: M_{t} \leq m^{[s]}, t \in T\right\}, M=\inf \left\{m_{t}: m_{t} \geq M^{[s]}, t \in T\right\}$, then

$$
\mathcal{M}^{[r]}(x, \Phi) \leq\left(\int_{T} \Phi_{t}\left(x_{t}^{r}\right) d \mu-\delta_{s, r} \widetilde{x}_{s}\right)^{1 / r} \leq \mathcal{M}^{[s]}(x, \Phi),
$$

where $\delta_{s, r} \geq 0$ for $r \leq-1, \delta_{s, r} \leq 0$ for $r \geq 1$ and $\widetilde{x}_{s} \geq 0$. Here we assume that $\delta_{s, r} \equiv \delta_{s, r}(\bar{m}, \bar{M}), \widetilde{x}_{s} \equiv \widetilde{x}_{s}(\bar{m}, \bar{M})$ are defined by (29) and $\bar{m} \in\left[m, m^{[s]}\right], \bar{M} \in\left[M^{[s]}, M\right], \bar{m}<\bar{M}$, are arbitrary numbers.

In the proof we use the same technique as in the proof of Corollary 3.5. We omit it.

Figure 4 shows regions (1),(2),(4),(6),(7) in which the monotonicity of the power mean holds true (see Corollary 2.4), also Figure 4 shows regions (1)-(7) which this holds true with condition on spectra (see Corollary 3.5). We show in [10, Example 2] that the order among power means does not hold generally without a condition on spectra in regions (3),(5). Now, by using Corollary 4.5 we obtain a refined inequality in the regions (2)-(6) (see Corollary 4.6).

Corollary 4.6. Let $\left(x_{t}\right)_{t \in T},\left(\Phi_{t}\right)_{t \in T}$ be as in the definition of the power mean (3). Let $m_{t}$ and $M_{t}, 0<m_{t} \leq M_{t}$ be the bounds of $x_{t}, t \in T$. Let

$$
\begin{array}{lll}
\left(m^{[r]}, M^{[r]}\right) \cap\left[m_{t}, M_{t}\right]=\varnothing, & t \in T, & m_{[r]}<M_{[r]}, \\
\left(m^{[s]}, M^{[s]}\right) \cap\left[m_{t}, M_{t}\right]=\varnothing, & t \in T, & m_{[s]}<M_{[s]}
\end{array}
$$




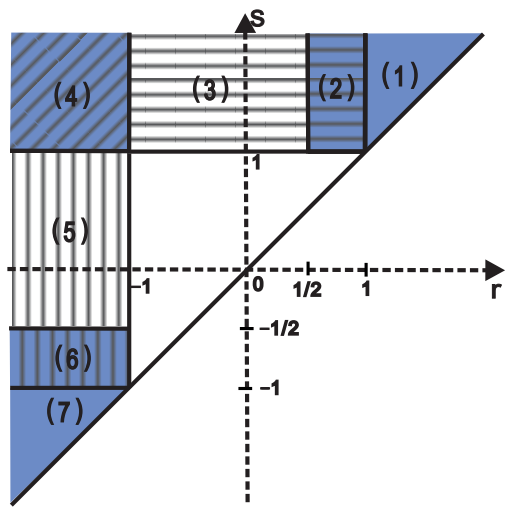

$M^{[r]}(\mathbf{x}, \Phi) \leq M^{[s]}(\mathbf{x}, \Phi) \quad$ in $\quad(1),(2),(4),(6),(7)$

WITHOUT CONDITION ON SPECTRA

$M^{[]]}(\mathbf{x}, \Phi) \leq M^{[r]}(\mathbf{x}, \Phi)+\Delta(r, s, \mathbf{x}) \leq M^{[s]}(\mathbf{A}, \Phi)$

in (2), (3), (4) or (4), (5), (6)

WITH CONDITION ON SPECTRA

Figure 4: Regions describing inequalities among power means

where $m^{[r]}, M^{[r]}, m^{[r]} \leq M^{[r]}$ and $m^{[s]}, M^{[s]}, m^{[s]} \leq M^{[s]}$ are the bounds of $\mathcal{M}^{[r]}(x, \Phi)$ and $\mathcal{M}^{[s]}(x, \Phi)$, respectively, and

$$
\begin{aligned}
& m_{[r]}=\max \left\{M_{t}: M_{t} \leq m^{[r]}, t \in T\right\}, \quad M_{[r]}=\min \left\{m_{t}: m_{t} \geq M^{[r]}, t \in T\right\}, \\
& m_{[s]}=\max \left\{M_{t}: M_{t} \leq m^{[s]}, t \in T\right\}, \quad M_{[s]}=\min \left\{m_{t}: m_{t} \geq M^{[s]}, t \in T\right\} .
\end{aligned}
$$

Let $\bar{m} \in\left[m_{[r]}, m^{[r]}\right], \bar{M} \in\left[M^{[r]}, M_{[r]}\right], \bar{m}<\bar{M}$, and $\overline{\bar{m}} \in\left[m_{[s]}, m^{[s]}\right], \overline{\bar{M}} \in\left[M^{[s]}, M_{[s]}\right], \overline{\bar{m}}<\overline{\bar{M}}$ be arbitrary numbers.

$$
\begin{aligned}
& \text { If } r \leq 1 \leq s, \text { then } \\
& \qquad \begin{aligned}
\mathcal{M}^{[r]}(x, \Phi) & \leq \mathcal{M}^{[r]}(x, \Phi)+\delta_{r, 1}(\overline{\bar{m}}, \bar{M}) \widetilde{x}_{r}(\bar{m}, \bar{M}) \\
& -\delta_{s, 1}(\overline{\bar{m}}, \bar{M}) \widetilde{x}_{s}(\overline{\bar{m}}, \overline{\bar{M}}) \leq \mathcal{M}^{[s]}(x, \Phi) .
\end{aligned} \\
& \text { If } r \leq-1 \leq s, \text { then } \\
& \qquad \begin{aligned}
\mathcal{M}^{[r]}(x, \Phi) & \leq \mathcal{M}^{[r]}(x, \Phi)+\left(\int_{T} \Phi_{t}\left(x_{t}^{-1}\right) d \mu-\delta_{s,-1}(\overline{\bar{m}}, \overline{\bar{M}}) \widetilde{x}_{s}(\overline{\bar{m}}, \overline{\bar{M}})\right)^{-1} \\
& -\left(\int_{T} \Phi_{t}\left(x_{t}^{-1}\right) d \mu-\delta_{r,-1}(\bar{m}, \bar{M}) \widetilde{x}_{r}(\bar{m}, \bar{M})\right)^{-1} \leq \mathcal{M}^{[s]}(x, \Phi) .
\end{aligned}
\end{aligned}
$$

If $r \leq-1, s \geq 1$, then

$$
\begin{aligned}
\mathcal{M}^{[r]}(x, \Phi) & \leq \mathcal{M}^{[r]}(x, \Phi)+\mathcal{M}^{[1]}(\mathbf{x}, \Phi)-\delta_{s, 1}(\overline{\bar{m}}, \overline{\bar{M}}) \widetilde{x}_{s}(\overline{\bar{m}}, \overline{\bar{M}}) \\
& -\left(\int_{T} \Phi_{t}\left(x_{t}^{-1}\right) d \mu-\delta_{r,-1}(\bar{m}, \bar{M}) \widetilde{A}_{r}(\bar{m}, \bar{M})\right)^{-1} \leq \mathcal{M}^{[s]}(x, \Phi) .
\end{aligned}
$$

The proof is similar to that of Corollary 4.4 and we omit it.

\section{References}

[1] M. Anwar, J. Pečarić, Means of the Cauchy Type, LAP Lambert Academic Publishing, 2009.

[2] J.I. Fujii, Path of quasi-means as a geodesic, Linear Algebra Appl. 434 (2011) 542-558.

[3] J.I. Fujii, M. Nakamura, S.-E. Takahasi, Coopers approach to chaotic operator means, Sci. Math. Jpn. 63 (2006) 319-324.

[4] T. Furuta, J. Mićić Hot, J. Pečarić, Y. Seo, Mond-Pečarić method in Operator Inequalities, Monographs in Inequalities 1, Element, Zagreb, 2005.

[5] J. Haluska, O. Hutnik, Some inequalities involving integral means, Tatra Mt. Math. Publs. 35 (2007) 131-146.

[6] F. Hansen, J. Pečarić, I. Perić, Jensen's operator inequality and its converses, Math. Scand. 100 (2007) 61-73.

[7] A. Kolesárová, Limit properties of quasi-arithmetic means, Fuzzy Sets and Systems 124 (2001) 65-71.

[8] F. Kubo, T. Ando, Means of positive linear operators, Math. Ann. 246 (1980) 205-224.

[9] J.-L. Marichal, On an axiomatization of the quasi-arithmetic mean values without the symmetry axiom, Aequationes Math. 59 (2000) 74-83. 
[10] J. Mićić, Z. Pavić, J. Pečarić, Jensen's inequality for operators without operator convexity, Linear Algebra Appl. 434 (2011) $1228-1237$.

[11] J. Mićić, Z. Pavić and J. Pečarić, Order among power operator means with condition on spectra, Math. Inequal. Appl. 14 (2011) 709-716.

[12] J. Mićić, Z. Pavić, J. Pečarić, Extension of Jensen's operator inequality for operators without operator convexity, Abstr. Appl. Anal. 2011 (2011) 1-14.

[13] J. Mićić, Z. Pavić, J. Pečarić, Some better bounds in converses of the Jensen operator inequality, Oper. Matrices, to appear.

[14] J. Mićić, J. Pečarić, J. Perić, Refined Jensen's operator inequality with condition on spectra, Oper. Matrices, to appear.

[15] J. Mićíć, J. Pečarić, J. Perić, Extension of the refined Jensen's operator inequality with condition on spectra, Ann. Funct. Anal., to appear.

[16] J. Mićić, J. Pečarić, Y. Seo, Converses of Jensen's operator inequality, Oper. Matrices 4 (2010) 385-403.

[17] J. Mićić, J. Pečarić, Y. Seo, Order among quasi-arithmetic means of positive operators, Math. Reports 13 (2012), in print.

[18] J.Mićić, J.Pečarić, Y.Seo, Function order of positive operators based on the Mond-Pečarić method, Linear Algebra Appl. 360 (2003) $15-34$.

[19] D.S. Mitrinović, J. Pečarić, A M. Fink, Classical and new Inequalities in Analysis, Kluwer Acad. Publ., Dordrecht-Boston-London, 1993.

[20] D.S. Mitrinović (with P.M. Vasić), Analytic Inequalities, Springer-Verlag, Berlin, 1970.

[21] M.S. Moslehian, An operator extension of the parallelogram law and related norm inequalities, Math. Inequal. Appl. 14 (2011) $717-725$. 